\title{
Pharmacological inhibition of EZH2 combined with DNA-damaging agents interferes with the DNA damage response in MM cells
}

\author{
LI XU $^{1 *}$, HAILONG TANG $^{1 *}$, KAI WANG $^{2 *}$, YANHUA ZHENG $^{1}$, JUAN FENG $^{1}$, \\ HONGJUAN DONG $^{1}$, YULONG JIN ${ }^{1}, \mathrm{CHUN} \mathrm{CAO}^{1}$, XIEQUN CHEN ${ }^{1}$ and GUANGXUN GAO ${ }^{1}$ \\ ${ }^{1}$ Department of Hematology, People's Liberation Army Center of Hematologic Disorders, Xijing Hospital, \\ Air Force Medical University, Xi'an, Shaanxi 710032; ${ }^{2}$ Department of Gastroenterology, \\ The 16th Hospital of The People's Liberation Army of China, Xinjiang 836599, P.R. China
}

Received May 2, 2018; Accepted December 13, 2018

DOI: $10.3892 / \mathrm{mmr} .2019 .10075$

\begin{abstract}
Enhancer of zeste homolog 2 (EZH2) serves a pivotal role in epigenetic silencing by acting as a histone methyltransferase. It has been confirmed that EZH 2 overexpression occurs in different types of cancer and is involved in drug resistance, while it remains unclear how a DNA-damaging event may promote EZH2 expression in multiple myeloma (MM) cells and how EZH2 influences its susceptibility to death in response to DNA-damaging chemotherapy. The present study examined the impact of EZH2 inhibition on DNA damage-induced apoptosis in MM cells and elucidated its underlying molecular mechanism. It was demonstrated that pharmacological inhibition of EZH2 sensitized MM cells to DNA-damaging agents and promoted limited caspase-dependent apoptosis. Mechanistically, targeting EZH2 with minimal toxic concentrations of a pharmacological inhibitor (GSK126) markedly weakened the accompanying increase in the histone trimethylation $\mathrm{H} 3 \mathrm{~K} 27 \mathrm{me} 3$ and aggravated DNA damage response (DDR)-associated apoptosis in vitro. These data preliminarily confirmed the underlying molecular mechanisms of interaction between histone methylation and the DDR in MM cells, forming the rationale for the combination regimen of EZH2 inhibitors with DNA-damaging agents for the treatment of MM.
\end{abstract}

Correspondence to: Dr Guangxun Gao or Dr Xiequn Chen, Department of Hematology, People's Liberation Army Center of Hematologic Disorders, Xijing Hospital, Air Force Medical University, 127 Changle Western Road, Xi'an, Shaanxi 710032, P.R. China

E-mail: gaoguangxun@fmmu.edu.cn

E-mail: xiequnchen@fmmu.edu.cn

*Contributed equally

Key words: enhancer of zeste homolog 2, DNA damage, serine-protein kinase ATM, multiple myeloma, chemotherapy sensitivity

\section{Introduction}

Multiple myeloma (MM) is a plasma cell malignancy for which there is currently no cure (1). Although the application of novel drugs with different mechanisms has notably improved the survival of patients with MM, the majority of patients eventually relapse, highlighting the need for additional agents with novel mechanisms. At present, DNA-damaging agents remain an important part of the first-line treatment of MM. The PAD regimen of bortezomib combined with doxorubicin and dexamethasone is associated with an excellent anti-MM effect, and its first-line status remains unquestionable (2). The combination of DNA-damaging agents with novel drugs is also an important direction for the treatment of MM.

Aberrations in epigenetic events are important during the development of MM; thus, the interruption of these processes may serve as an efficacious anti-MM strategy $(3,4)$. It has been well acknowledged that polycomb group (PcG) proteins take an active part in silencing homeotic genes during embryonic development (5). At the molecular level, PcG proteins are classified into two groups, termed polycomb repressive complexes (PRC1 and PRC2). Enhancer of zeste homolog 2 (EZH2) interacts with other PcG proteins, including suppressor of zeste 12 and embryonic ectoderm development, all of which compose PRC2 (5). It has been established that overexpression of EZH2 is frequently observed in a variety of human malignancies, including MM, and is associated with poor prognosis (6). Moreover, EZH2 epigenetically represses the expression of tumor suppressor genes through trimethylation of histone H3K27 to mediate cell proliferation, invasion and metastasis (7). Thus, it appears that targeting EZH2 may be applicable to the treatment of MM.

A previous study reported that $\mathrm{PcG}$ proteins are not only involved in epigenetic gene silencing, but also modulate the outcomes of cancer cells in response to DNA damage (8). Specifically, PcG proteins have been demonstrated to accumulate in areas of DNA double strands breaks (DSBs). This is substantiated by the fact that the loss of PcG genes decreases the ability of cells to repair DSBs and renders them sensitive to ionizing radiation (9). However, the involvement of EZH2 
in the cellular response to DNA damage in MM cells has not been extensively investigated.

The present study aimed to investigate the impact of EZH2 inhibition on the anti-MM potency of the DNA-damaging agents doxorubicin (DOX)/melphalan (MEL), and to preliminarily discuss the possible synergistic mechanism from the perspectives of epigenetics and the DNA damage response (DDR), with the intention of laying a foundation for an improved understanding of the regulatory mechanism of $\mathrm{EZH} 2$ in MM, and a rationale for developing novel therapeutic strategies for MM.

\section{Materials and methods}

Cell culture and reagents. The human MM cell lines RPMI8226 and H929 (American Type Culture Collection, Manassas, VA, USA) were cultured under typical conditions $\left(37^{\circ} \mathrm{C}\right.$ with $5 \% \mathrm{CO}_{2}$ ) in RPMI-1640 medium (HyClone; GE Healthcare Life Sciences, Logan, UT, USA) supplemented with $10 \%$ fetal bovine serum (Zeta-Life Company, San Francisco, CA, USA), penicillin $(100 \mathrm{U} / \mathrm{ml})$, and streptomycin $(100 \mu \mathrm{g} / \mathrm{ml})$. DOX and MEL (Sigma-Aldrich; Merck KGaA, Darmstadt, Germany) were kept in dimethyl sulfoxide (DMSO) at 50 and $10 \mathrm{mM}$ stock concentrations, respectively. GSK126 was kept in DMSO at a $50 \mathrm{mM}$ stock concentration. KU-55933 was kept in DMSO at a $50 \mathrm{mM}$ stock concentration.

Cell proliferation assay. Cells were seeded into 96-well plates at $1.5 \times 10^{4}$ cells/well for $48 \mathrm{~h}$. MTT (Sigma-Aldrich; Merck $\mathrm{KGaA}$ ) was used to determine cell proliferation. Cells were incubated in $10 \% \mathrm{MTT}$ reagent at $37^{\circ} \mathrm{C}$ for $4 \mathrm{~h}$ and dissolved in $150 \mu \mathrm{l}$ DMSO (Sigma-Aldrich; Merck KGaA) for $10 \mathrm{~min}$. Subsequently, the absorbance at $490 \mathrm{~nm}$ was measured using a microplate reader. All the experiments were performed three times.

Cell cycle and apoptosis assay. For the cell cycle assay, cells were gathered at $48 \mathrm{~h}$, washed in ice-cold PBS and fixed in ice-cold $70 \%$ ethanol overnight. Following removal of the ethanol, the cells were stained in the dark with $50 \mu \mathrm{g} / \mathrm{ml}$ propidium iodide (PI; Sigma-Aldrich; Merck KGaA) and $100 \mu \mathrm{g} / \mathrm{ml}$ RNase for $30 \mathrm{~min}$ at room temperature. The stained cells were examined for DNA content by Epics XL flow cytometry (Beckman Coulter, Inc., Brea, CA, USA).The result was analyzed by ModFit LT software (version 3.1, Verity Software House Inc., Topsham, ME, USA).

To identify apoptosis, flow cytometry analysis was performed with an Annexin V-Phycoerythrin (PE) Apoptosis Detection kit (BD Biosciences, San Jose, CA, USA). Cells were stained with $5 \mu 1$ Annexin V-PE, $5 \mu 1$ 7-aminoactinomycin D (AAD), and $500 \mu 11 \mathrm{X}$ binding buffer for $15 \mathrm{~min}$, and subsequently examined by Epics XL flow cytometry. The result was analyzed by CXP software (version 2.1, Beckman Coulter, Inc., Brea, CA, USA).

Western blotting. Cells were harvested and lysed with radioimmunoprecipitation assay buffer (Beyotime Institute of Biotechnology, Haimen, China) for $10 \mathrm{~min}$ on ice. Supernatant from every tube was gathered and moved to a new tube. The concentration of proteins in cell lysates was measured using a BCA assay. Identical amounts $(20 \mu \mathrm{g})$ of protein lysates were separated on $8-15 \%$ by SDS-PAGE gels (Bio-Rad Laboratories, Inc., Hercules, CA, USA) and transferred to a polyvinylidene fluoride membrane (EMD Millipore, Billerica, MA, USA). Membranes were blocked in 5\% skim milk for $1 \mathrm{~h}$ at room temperature. Membranes were incubated with primary antibodies against cleaved caspase-3 (1:500; cat. no. 9664; Cell Signaling Technology, Boston, MA, USA ), serine-protein kinase ATM (ATM; 1:5,000; cat. no. ab32420; Abcam, Cambridge, MA, USA), phosphorylated ATM (pATM; 1:5,000; cat. no. ab81292), EZH2 (1:1,000; cat. no. ab191080), cleaved poly (ADP-ribose) polymerase (cleaved PARP; 1:1,000; ab74290), cellular tumor antigen p53 (p53; 1:1,000; cat. no. ab32389), $\gamma$-histone H2AX ( $\gamma$-H2AX; 1:1,000; cat. no. ab2893), GAPDH (1:3,000; cat. no. CW0101; CWBIO, Beijing, China), H3K27me3 (1:10,000; cat. no. 07-449; Millipore, Temecula, CA, USA) and total histone $\mathrm{H} 3$ (1:5,000; cat. no. ab1791) at $4^{\circ} \mathrm{C}$ overnight. Membranes were washed 3 times with TBS-T and were incubated with peroxidase-conjugated goat anti-rabbit $\operatorname{IgG}(1: 4,000$; cat. no. ZB-2301, OriGene Technologies, Inc., Beijing, China) for $1 \mathrm{~h}$ at room temperature. Immunoblotting was detected using an Enhanced Chemiluminescence Western Blot kit (EMD Millipore). All western blotting data were confirmed in a minimum of three independent biological experiments.

Statistical analysis. GraphPad Prism 5 (GraphPad Software, Inc., La Jolla, CA, USA) was utilized for all the data analyses. Data are presented as the mean \pm standard deviation. Differences between multiple groups were confirmed using one-way analysis of variance followed by Tukey's post hoc test. The significance of differences between two groups was determined by Student's t-test. Data were obtained from at least three independent experiments. $\mathrm{P}<0.05$ was considered to indicate a statistically significant difference. The impacts of synergy and antagonism between GSK126 and DOX or MEL were examined using Calcusyn software (version 2.0; Biosoft, Great Shelford, UK). The combination index (CI) was calculated via the Chou-Talalay method (10). Drug synergism and antagonism were established by $\mathrm{CI}$, which quantitatively established additivity $(\mathrm{CI}=1)$, synergy $(\mathrm{CI}<1)$ and antagonism $(\mathrm{CI}>1)$. The resulting values were utilized in the construction of a plot of CI values over a range of affected fractions (Fa-CI plot) $(10,11)$.

\section{Results}

Pharmacological inhibition of EZH2 sensitizes MM cells to DNA-damaging agents. First, the present study assessed whether pharmacological inhibition of EZH2 with GSK126 was able to enhance DNA-damaging agent-induced synergistic lethality in MM cells in vitro. RPMI8226 and H929 cells were treated with DMSO, GSK126, DNA-damaging agents or combinations of GSK126 $(2.5,5$, and $10 \mu \mathrm{M})$ with DNA-damaging agents $(0.05,0.1$ or $0.2 \mu \mathrm{M}$ DOX; or 5,10 or $20 \mu \mathrm{M}$ of MEL) for $48 \mathrm{~h}$. Cell proliferation was assessed by MTT assay. The results indicated that the combination treatment yielded a stronger anti-proliferative effect compared with either drug alone. CI plot analysis using GSK126/DOX or GSK126/MEL at fixed concentration ratios of 1:50 and 1:2 indicated that co-treatment with GSK126 and DNA-damaging agents inhibited cell proliferation synergistically at the majority of concentrations, as indicated by the CI (Fig. 1). This experiment established the 

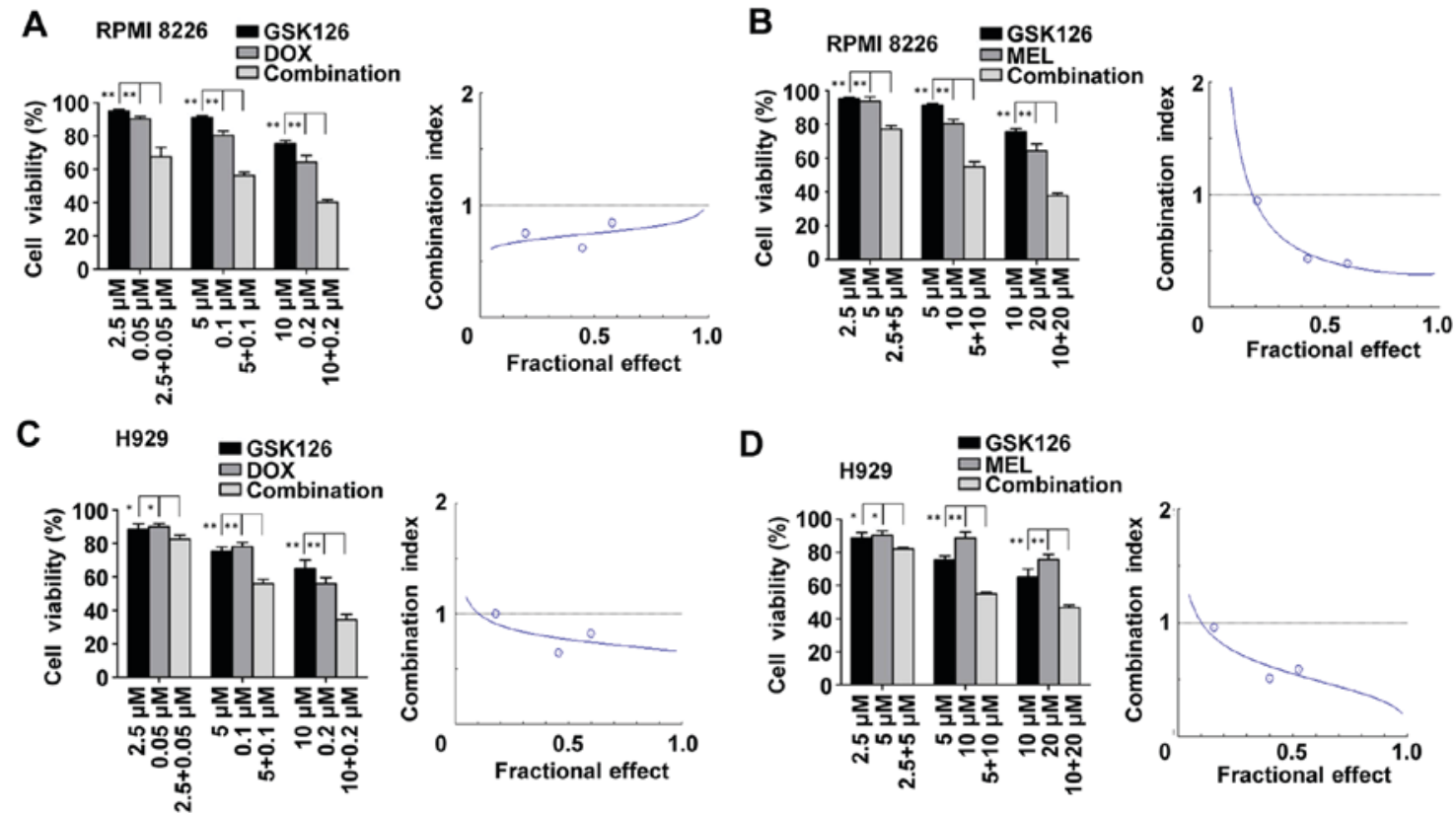

Figure 1. Enhancer of zeste homolog inhibitor GSK126 sensitizes MM cells to DOX- and MEL-induced cell death. (A) RPMI8226 cells were treated with DOX at the indicated concentrations in the presence or absence of GSK126 $(2.5,5$ and $10 \mu \mathrm{M})$ for $48 \mathrm{~h}$ and subjected to MTT-based assay. Dose-effect curves were produced using the commercially available software, Calcusyn 2.0. (B) RPMI8226 cells were treated with MEL at the indicated concentrations in the presence or absence of GSK126 $(2.5,5$ and $10 \mu \mathrm{M})$ for $48 \mathrm{~h}$ and subjected to MTT-based assay. Dose-effect curves were produced using the commercially available software, Calcusyn 2.0. (C) H929 cells were treated with DOX at the indicated concentrations in the presence or absence of GSK126 (2.5, 5 and $10 \mu \mathrm{M})$ for $48 \mathrm{~h}$ and subjected to MTT-based assay. Dose-effect curves were produced using the commercially available software, Calcusyn. (D) H929 cells were treated with MEL at the indicated concentrations in the presence or absence of GSK126 $(2.5,5$ and $10 \mu \mathrm{M})$ for $48 \mathrm{~h}$ and subjected to MTT-based assay. Dose-effect curves were produced using the commercially available software, Calcusyn 2.0. Data are representative of a minimum of three independent experiments. "P<0.05, ${ }^{* *} \mathrm{P}<0.01$. DOX, doxorubicin; MEL, melphalan.

phenomenon of the synergistic effect of combination GSK126 with DNA-damaging agents against MM cells. To examine the mechanism of action of GSK126 against MM cells in vivo, the concentration with the most pronounced synergistic effect was selected. Subsequent experiments in vitro were performed with GSK126 at $5 \mu \mathrm{M}$, which is below its half-maximal inhibitory concentration for each of the MM cell lines.

Pharmacological inhibition of EZH2 influences DNA-damaging agent-induced cell cycle redistribution. Cell cycle regulation serves an important role in cell proliferation; thus, the present study investigated whether functional inhibition of EZH2 affected cell cycle progression. Flow cytometry analysis following PI staining was used to assess the cell cycle distribution induced by GSK126, DNA-damaging agents or combination treatment. It was evident that EZH2 inhibition resulted in arrest at the G2/M phase and DNA-damaging agents induced G0/G1 phase exit in RPMI8226 and H929 cells (Fig. 2). Notably, combination treatment simultaneously induced G0/G1 and G2/M phase arrest in RPMI8226 and H929 cells (Fig. 2), suggesting that GSK126 and DNA-damaging agents might serve an independent role in cell cycle progression without crosstalk effects.

PharmacologicalinhibitionofEZH2 augmentsDNA-damaging agent-induced apoptosis in MM cells. To further illustrate the direction of cell fate that was triggered by these combination regimens, Annexin V-PE/7-AAD apoptosis detection was conducted to quantitatively determine the apoptosis of MM cells. The apoptosis-promoting effects were observed. GSK126 and DNA-damaging agents triggered an increase in apoptosis relative to the control group in RPMI8226 or H929 cells. Moreover, GSK126 markedly enhanced DNA-damaging agent-induced apoptosis compared with that of either single agent group (Fig. 3).

To further verify the apoptosis induced by single agents or combination treatment, the present study evaluated the expression of cleaved PARP, 553 and cleaved caspase- 3 proteins by western blotting performed on whole-cell lysates from control and treated MM cells, presented in Fig. 3. In accordance with the findings of the flow cytometry, the combination treatment increased the expression levels of cleaved PARP, p53 and cleaved caspase-3 compared with GSK126 and DNA-damaging agents alone in RPMI8226 and H929 cells. These results demonstrated that apoptosis was the principal fate of MM cells in the model of the synergistic inhibition of combined application of GSK126 and DNA-damaging agents, suggesting that EZH2 is an important factor affecting the cellular response to chromosomal lethality.

Pharmacological inhibition of EZH2 weakens the accompanying increase in $H 3 K 27 m e 3$ induced by DNA-damaging agents and further heightens DNA damage in this apoptotic model of MM cells. A number of previous studies have reported on the inextricable association between histone methylation and the DDR $(3,9,10)$. Treatment of MM cells with increasing concentrations of GSK126 for $48 \mathrm{~h}$ revealed decreases in H3K27me3 expression (Fig. 4A). EZH2 and H3K27me3 were upregulated following treatment with different concentrations of DNA-damaging agents over the course of $48 \mathrm{~h}$ in MM cells (Fig. 4B), suggesting that the 
A RPM18226
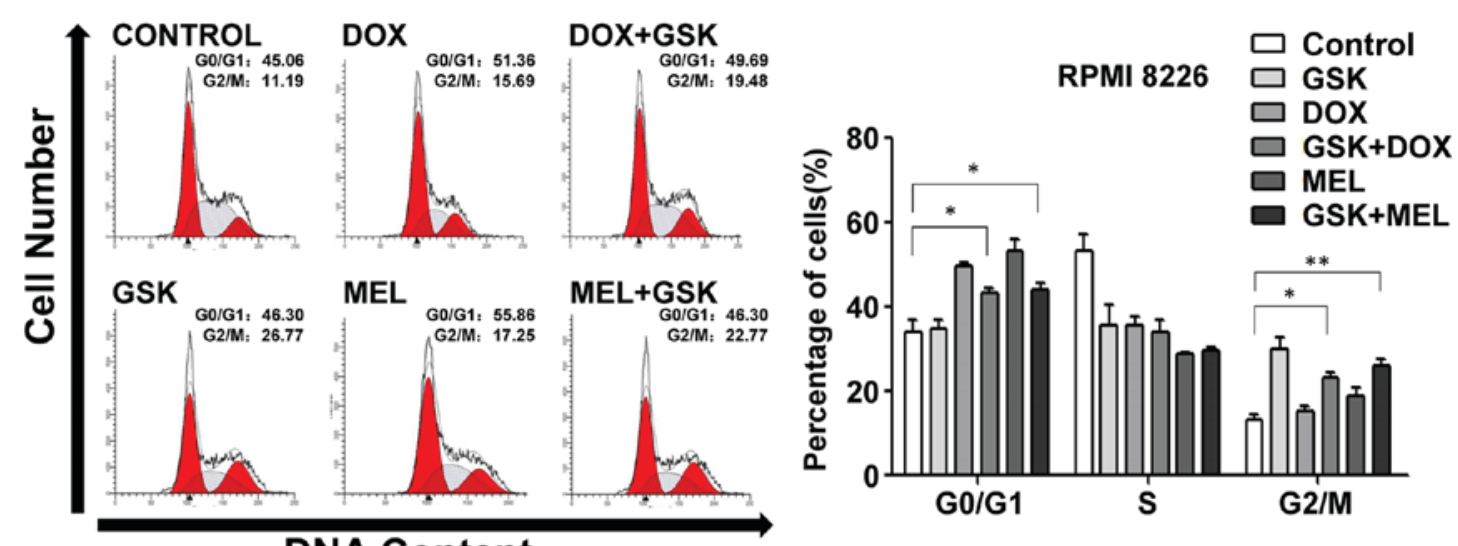

B

DNA Content
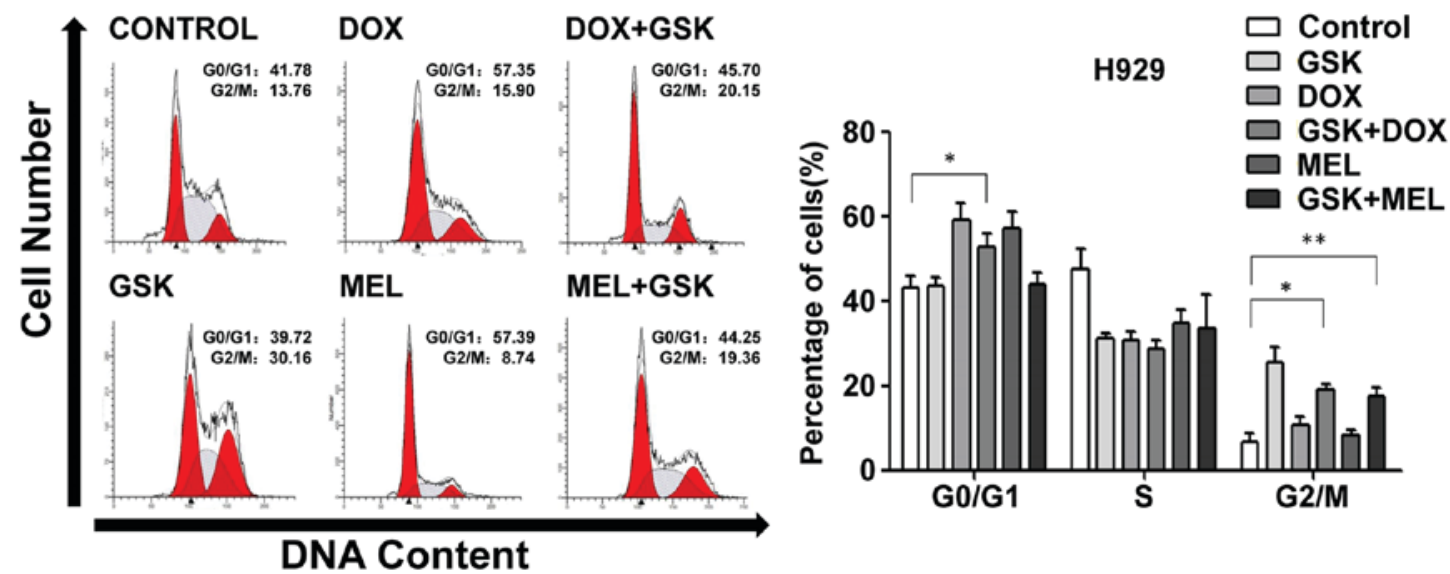

Figure 2. GSK 126 influences DNA-damaging agent-induced cell cycle redistribution. (A) RPMI8226 and (B) H929 cells were treated with GSK126 (5 $\mu$ M), DOX $(0.1 \mu \mathrm{M})$, MEL $(10 \mu \mathrm{M})$ or a combination treatment for $48 \mathrm{~h}$. Cell cycle distribution was established by flow cytometric evaluation. Data are representative of a minimum of three independent experiments. ${ }^{*} \mathrm{P}<0.05,{ }^{* *} \mathrm{P}<0.01$. DOX, doxorubicin; MEL, melphalan.

concomitant hypermethylation of histones following DNA damage may be involved in the DDR. To further understand the scope of the function of EZH2/H3K27me3 in the DDR, the present study focused on the alteration in the expression of EZH2/H3K27me3 and DDR components under combination treatment. The results revealed that in this synergistic inhibition model of MM cells, EZH2-inhibited cells exhibited a decrease in DNA-damaging agent-induced histone hypermethylation, and hyperactivation of DNA-damaging agent-induced ATM and H2AX phosphorylation (Fig. 4C). These results indicated that GSK126 likely amplifies DNA-damaging agent-triggered DNA damage signaling by generating histone hypomethylation, thereby serving a synergistic anti-MM role with DNA-damaging agents.

Inhibition of ATM activity interrupts ATM-dependent DNA damage signaling and caspase-3 cleavage. To thoroughly understand the mechanism by which GSK126 enhanced the anti-MM effect of DNA-damaging agents by affecting DNA damage signaling, the ATM inhibitor KU-55933 was added to suppress ATM activity in the synergistic effect model, followed by detection of the expression of proteins associated with DNA damage and cleaved caspase- 3 . The results demonstrated that the loss of ATM kinase activity impaired the upregulation of ATM phosphorylation and the expression of downstream $\gamma$-H2AX in this synergistic effect model, which resulted in a decrease in cleaved caspase-3, indicating the remission of apoptosis in MM cells (Fig. 5). These results further indicated that the synergistic anti-MM effect mediated by pharmacological inhibition of EZH2 was largely due to regulation of the ATM-dependent DDR.

\section{Discussion}

The incurability and likely recurrence of MM have driven the emergence of novel drugs in recent years; additionally, the appearance of more therapeutics also reflects the difficulty of finding a cure for MM $(11,12)$. The development of novel drugs for MM is ongoing. Studies have demonstrated that high expression of EZH2 in MM cells indicates a poor outcome, and targeting EZH2 may induce apoptosis in MM cells $(13,14)$. In this way, EZH2 is a promising drug target for MM therapy. The present study focused on EXH2 to examine the synergistic anti-MM effect of pharmacological inhibition of EZH2 with DNA-damaging agents, and the associated mechanisms were preliminarily discussed.

Growing evidence has also demonstrated that EZH2 is overexpressed in numerous cancer types, and its overexpression 
A

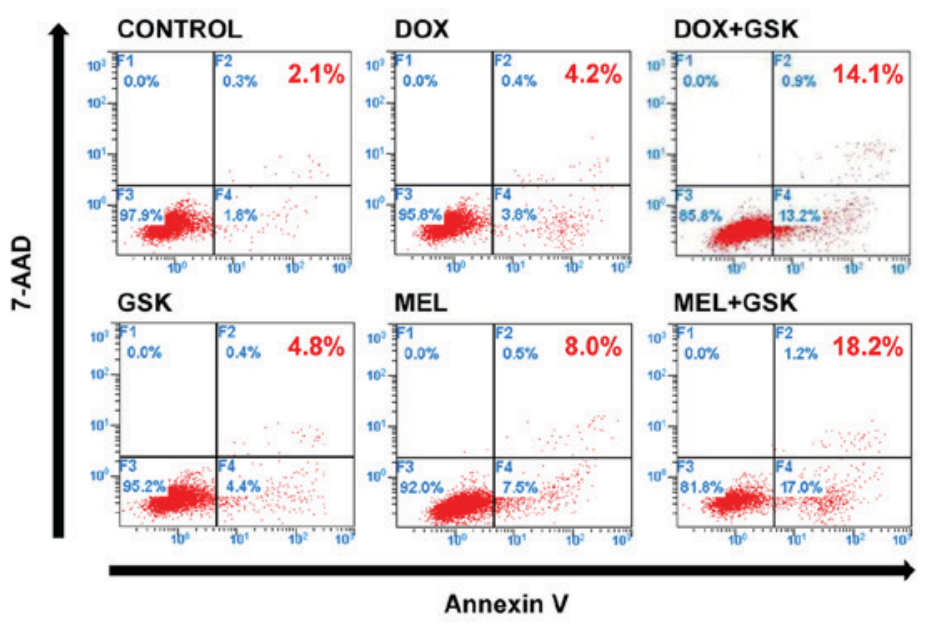

B

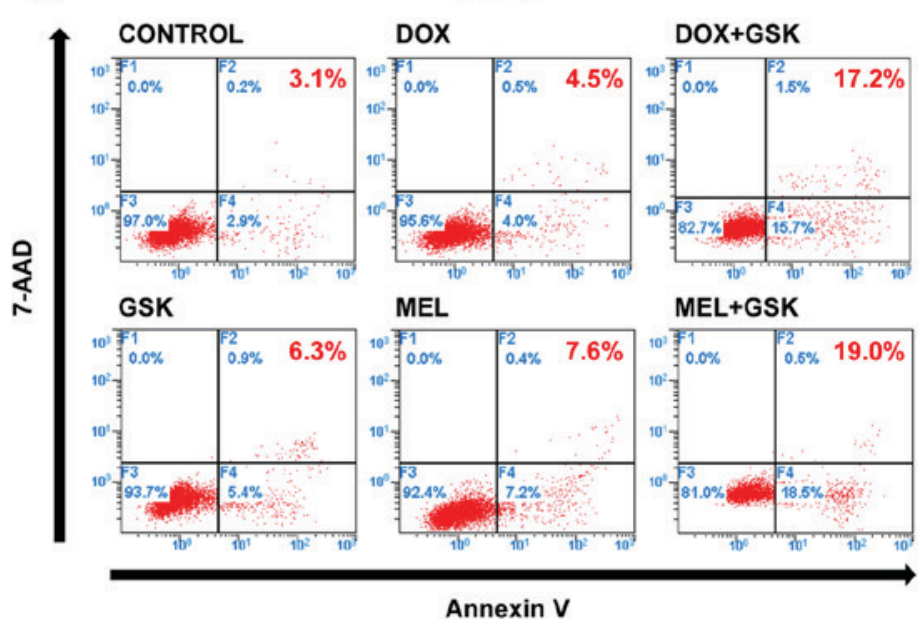

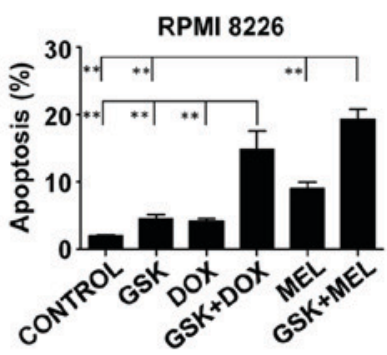

RPMI 8226
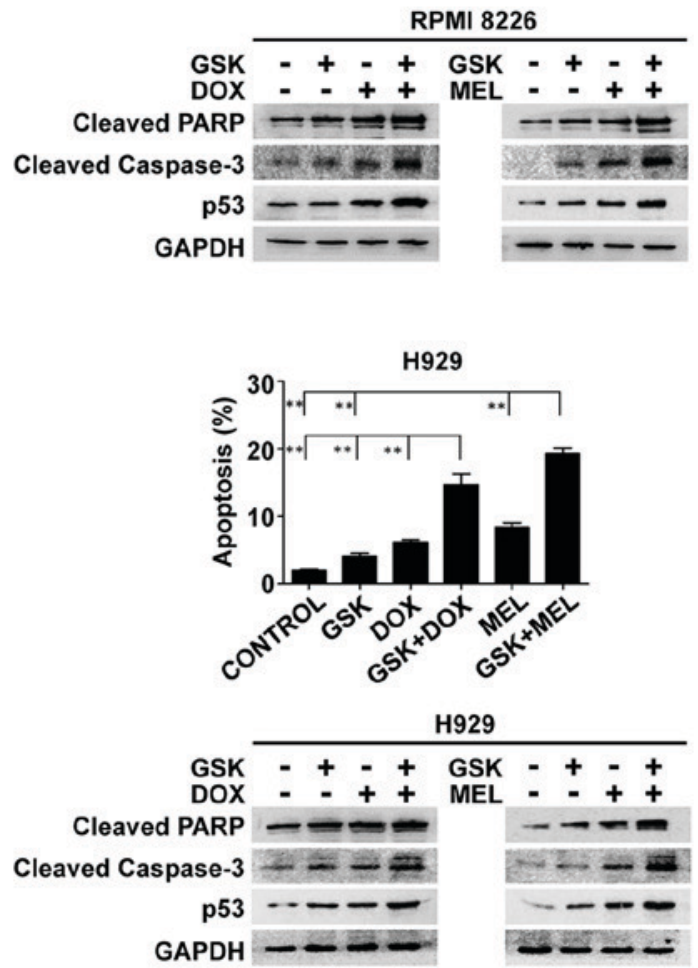

Figure 3. GSK 126 augments DNA-damaging agent-induced apoptosis in MM cells. (A) RPMI8226 and (B) H929 cells were treated with GSK126 (5 $\mu$ M), DOX $(0.1 \mu \mathrm{M}), \mathrm{MEL}(10 \mu \mathrm{M})$ or a combination treatment for $48 \mathrm{~h}$. The percentage of apoptotic cells was established by flow cytometry with Annexin V-phycoerythrin and 7-aminoactinomycin D staining. ${ }^{* *} \mathrm{P}<0.01$. Whole-cell lysates were utilized to evaluate the protein expression of cleaved PARP, cleaved caspase-3 and p53 via western blotting. GAPDH was utilized as the loading control. All the experiments represent the mean \pm standard deviation from three independent experiments. DOX, doxorubicin; MEL, melphalan; PARP, poly (ADP-ribose) polymerase; p53, cellular tumor antigen p53.

is associated with cancer resistance and poor prognosis. Due to the role of epigenetic regulators in gene silencing and chromatin structure modulation, studies have identified a function for PcG proteins in the cellular response to DNA damage that indicates that $\mathrm{PcG}$ genes directly and indirectly control different aspects of the DDR (15-17). The PcG gene EZH2 may impact DSB repair indirectly via suppression of the homologous recombination enzyme DNA repair protein RAD51 homolog 1 and of its paralogs, and through the modulation of breast cancer type 1 susceptibility protein in human uterine fibroids (18). Meanwhile, it remains unknown how EZH2 mediates DDR signaling and what its role in transcriptional silencing at DNA breaks in MM cells may be, thus epigenetic regulation may effectively enhance the anti-MM effect of DNA-damaging agents. The present results confirmed this conjecture that GSK126, an inhibitor of EZH2, combined with DNA-damaging agents synergistically induced apoptosis in MM cells.

It was observed that DOX and MEL induced upregulation of EZH2 and its downstream molecule H3K27me3. Combining an EZH2 inhibitor with DOX or MEL triggered the hyperphosphorylation of ATM and H2AX and further directed DNA damage by amplifying the apoptotic response. The results demonstrated that ATM inactivity induced by KU-55933 interrupted ATM-dependent DNA damage signaling and caspase-3 cleavage, further indicating that the synergistic anti-MM effect mediated by the pharmacological inhibition of EZH2 was at least partially due to the ATM-dependent DDR. These results were consistent with a previous report that EZH2 inhibition by DZNep or GSK126, combined with other DNA-damaging agents (including etoposide or cisplatin), modifies the cellular response to apoptosis (19).

It is known that the DNA damage reaction consists of the identification of the DNA lesion, transduction of the damage signal, and the establishment of conditions that induce DNA repair (20). The DDR-associated protein ATM detects DSBs and stimulates the DNA-damage checkpoint, causing cell cycle arrest and an increase in the concentration of proteins associated with the repair of DNA damage to maintain the stability of the genome (21). It was hypothesized that EZH2 may be involved in DNA damage repair and influence the accumulation of other key 
A

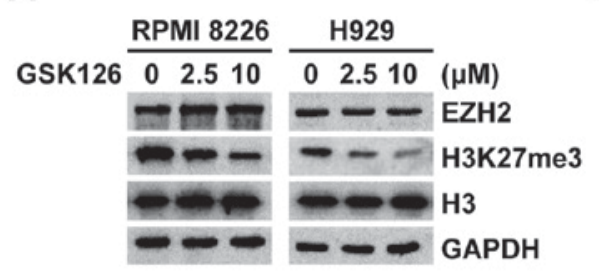

B

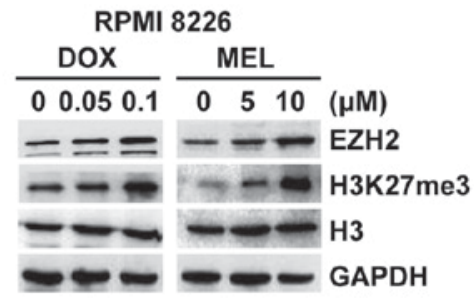

H929

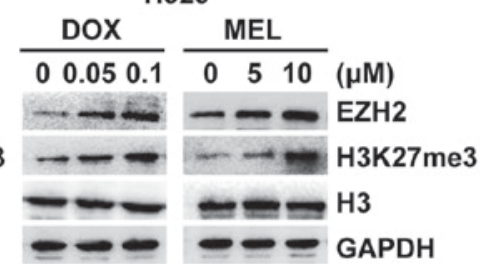

C

RPMI 8226
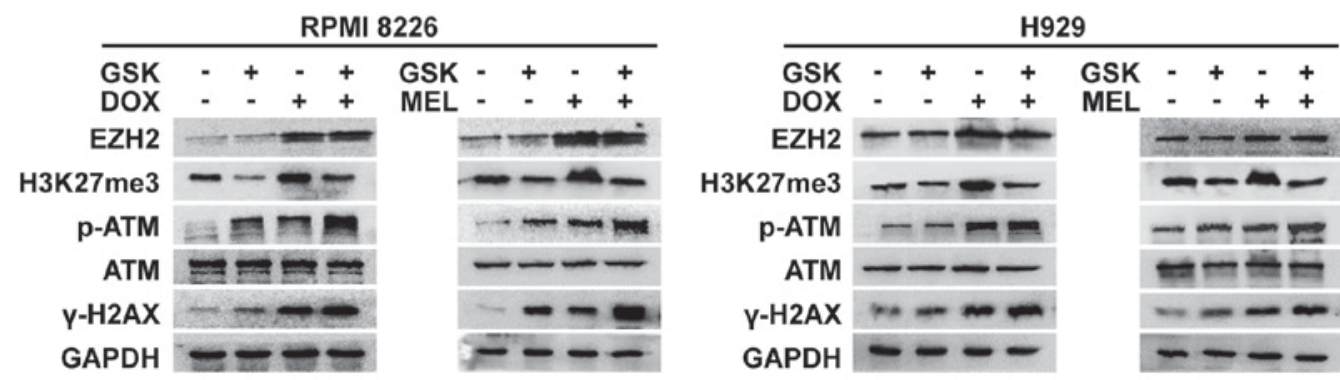

Figure 4. GSK126 weakens the accompanying increase in H3K27me3 induced by DNA-damaging agents, and further heightens DNA damage in this apoptotic model of MM cells. (A) RPMI8226 and H929 cells were treated with different concentrations of GSK126 for $48 \mathrm{~h}$. Whole-cell lysates were utilized to evaluate EZH2 and H3K27me3. Total histone H3 and GAPDH were utilized as the loading control. (B) RPMI8226 and H929 cells were treated with different concentrations of DNA-damaging agents for $48 \mathrm{~h}$. Whole-cell lysates were utilized to evaluate EZH2 and H3K27me3. Total histone H3 and GAPDH were utilized as the loading control. (C) RPMI8226 and H929 cells were treated with GSK126 (5 $\mu \mathrm{M})$, DOX $(0.1 \mu \mathrm{M})$, MEL $(10 \mu \mathrm{M})$ or a combination treatment for $48 \mathrm{~h}$. Whole-cell lysates were utilized to evaluate EZH2, H3K27me3, p-ATM, ATM and $\gamma$-H2AX. GAPDH served as the loading control. DOX, doxorubicin; MEL, melphalan; EZH2, enhancer of zeste homolog 2; p, phosphorylated; ATM, serine-protein kinase ATM; H2AX, histone H2AX; H3K27me3, trimethylated histone $\mathrm{H} 3 \mathrm{~K} 27$.

\section{A}

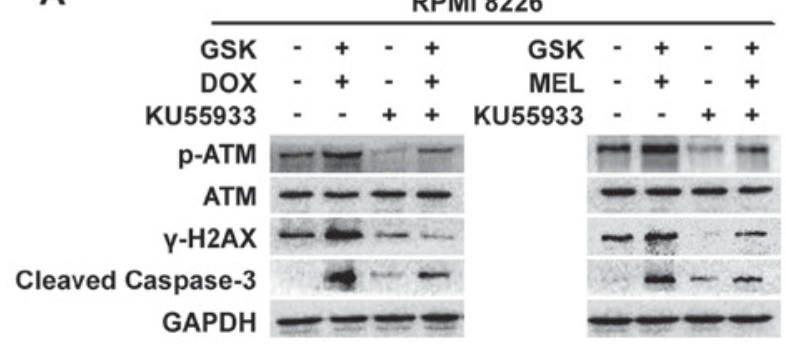

B

\begin{tabular}{|c|c|c|c|c|c|c|}
\hline \multicolumn{6}{|c|}{ H929 } & \\
\hline GSK & $-\quad+$ & + & GSK & - & & + \\
\hline DOX & $-\quad+$ & $-\quad+$ & MEL & $-\quad+$ & & \\
\hline KU55933 & - & ++ & KU55933 & $-\quad-$ & & \\
\hline p-ATM & $=$ & $n=$ & & -- & & - \\
\hline ATM & -- & -- & & -- & & - \\
\hline $\mathrm{y}-\mathrm{H} 2 \mathrm{AX}$ & $-\infty$ & $=-1=$ & & -- & - & - \\
\hline sppase-3 & -- & 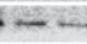 & & -8 & & \\
\hline DH & - & - & & $-=$ & & \\
\hline
\end{tabular}

Figure 5. Inhibition of ATM activity interrupts ATM-dependent DNA damage signaling and caspase-3 cleavage. (A) RPMI8226 and (B) H929 cells were treated with KU-55933 $(10 \mu \mathrm{M})$, GSK126 with DNA-damaging agents or a combination treatment for $48 \mathrm{~h}$. Whole-cell lysates were utilized to evaluate p-ATM, ATM, $\gamma$-H2AX and cleaved caspase-3. GAPDH served as the loading control. p, phosphorylated; ATM, serine-protein kinase ATM; H2AX, histone H2AX; DOX, doxorubicin; MEL, melphalan.

DDR proteins to the DNA damage foci. GSK126 alters the chromatin status and genomic stability, which subsequently affects the initiation and amplification of DDR signaling in addition to the accompanying DNA repair (22). The inhibition of EZH2 may cause a decrease in DNA repair activity, leading to more marked cell apoptosis. Another possibility for ATM signaling activation via EZH2 inhibition is the induction of oxidative stress. Based on previous studies, mitochondrial damage is substantially increased by a combination of etoposide and GSK126. It is also associated with EZH2-H3K27me3-induced gene silencing of Bcl-2 homologous antagonist/killer and apoptosis regulator BAX $(23,24)$. The inhibition of EZH2 leads to microRNA (miR)-29b upregulation together with the suppression of miR-29b downstream pro-survival targets, including SP1, MCL-1 and cyclin-dependent kinase 6. Inhibition of miR-29b markedly reduces the sensitivity of MM cells to small molecule EZH2-inhibitors indicating that tumor suppressor miRs may also be involved in epigenetic alterations (25). However, the precise mechanism underlying GSK126-induced ATM activation in MM cells is beyond the scope of the present study, and future studies are required.

In conclusion, the present study preliminarily demonstrated that pharmacological inhibition of EZH2 by GSK126 enhanced the anti-MM effect of DNA-damaging agents, partially by decreasing $\mathrm{H} 3 \mathrm{~K} 27 \mathrm{me} 3$ and via involvement in the regulation of the ATM-dependent DDR. With the toxic effect of chemotherapy, any sensitizer that is able to effect even a mild DNA damage reaction, triggering an apoptotic process, may be able to improving the efficacy of DNA-damaging chemotherapeutic agents and limit their toxic effects (26). The present study ultimately presented a novel and effective method of treating patients with MM.

\section{Acknowledgements}

Not applicable. 


\section{Funding}

This study was supported by the Social Development Science and Technology Fund of Shaanxi Province (grant no. 2016SF-071) and the Natural Science Foundation of Shaanxi Province (grant no. 2017JM8025).

\section{Availability of data and materials}

All data generated or analyzed in this study are included in this manuscript.

\section{Authors' contributions}

GG and XC conceived and designed the experiment. LX and HT designed and performed the experiments, analyzed the data and drafted the manuscript. $\mathrm{KW}, \mathrm{YZ}$ and JF performed the experiments and analyzed the data. HD, YJ and CC acquired and interpreted the data. GG gave final approval of the article. All authors have reviewed the final version of the manuscript and approved it for publication.

\section{Ethics approval and consent to participate}

Not applicable.

\section{Patient consent for publication}

Not applicable.

\section{Competing interests}

The authors declare that they have no competing interests.

\section{References}

1. Pourabdollah $\mathrm{M}$ and Chang $\mathrm{H}$ : Coexistence of multiple myeloma and Paget disease of bone. Blood 130: 1173, 2017.

2. Mai EK, Bertsch U, Durig J, Kunz C, Haenel M, Blau IW, Munder M, Jauch A, Schurich B, Hielscher T, et al: Phase III trial of bortezomib, cyclophosphamide and dexamethasone (VCD) versus bortezomib, doxorubicin and dexamethasone (PAd) in newly diagnosed myeloma. Leukemia 29: 1721-1729, 2015.

3. Issa ME, Takhsha FS, Chirumamilla CS, Perez-Novo C, Vanden Berghe $\mathrm{W}$ and Cuendet $\mathrm{M}$ : Epigenetic strategies to reverse drug resistance in heterogeneous multiple myeloma. Clin Epigenetics 9: 17, 2017.

4. Pawlyn C, Kaiser MF, Davies FE and Morgan GJ: Current and potential epigenetic targets in multiple myeloma. Epigenomics 6: 215-228, 2014

5. Sanulli S, Justin N, Teissandier A, Ancelin K, Portoso M, Caron M, Michaud A, Lombard B, da Rocha ST, Offer J, et al: Jarid2 methylation via the PRC2 complex regulates H3K27me3 deposition during cell differentiation. Mol Cell 57: 769-783, 2015.

6. Pawlyn C, Bright MD, Buros AF, Stein CK, Walters Z, Aronson LI, Mirabella F, Jones JR, Kaiser MF, Walker BA, et al: Overexpression of EZH2 in multiple myeloma is associated with poor prognosis and dysregulation of cell cycle control. Blood Cancer J 7: e549, 2017.

7. Cao R, Wang L, Wang H, Xia L, Erdjument-Bromage H, Tempst P, Jones RS and Zhang Y: Role of histone H3 lysine 27 methylation in Polycomb-group silencing. Science 298 1039-1043, 2002.

8. Ito T, Teo YV, Evans SA, Neretti N and Sedivy JM: Regulation of cellular senescence by polycomb chromatin modifiers through distinct DNA damage- and histone methylation-dependent pathways. Cell Rep 22: 3480-3492, 2018.
9. Zeidler M, Varambally S, Cao Q, Chinnaiyan AM, Ferguson DO, Merajver SD and Kleer CG: The polycomb group protein EZH2 impairs DNA repair in breast epithelial cells. Neoplasia 7: 1011-1019, 2005.

10. Ha K, Lee GE, Palii SS, Brown KD, Takeda Y, Liu K, Bhalla KN and Robertson KD: Rapid and transient recruitment of DNMT1 to DNA double-strand breaks is mediated by its interaction with multiple components of the DNA damage response machinery. Hum Mol Genet 20: 126-140, 2011.

11. Kuroda J: Therapeutic approach for relapsed/refractory multiple myeloma: The logic and practice. Rinsho Ketsueki 58: 2058-2066, 2017 (In Japanese).

12. Varga C, Maglio M, Ghobrial IM and Richardson PG: Current use of monoclonal antibodies in the treatment of multiple myeloma. Br J Haematol 181: 447-459, 2018.

13. Kuser-Abali G, Gong L, Yan J, Liu Q, Zeng W, Williamson A, Lim CB, Molloy ME, Little JB, Huang L and Yuan ZM: An EZH2-mediated epigenetic mechanism behind p53-dependent tissue sensitivity to DNA damage. Proc Natl Acad Sci USA 115: 3452-3457, 2018.

14. Wang J, Cheng P, Pavlyukov MS, Yu H, Zhang Z, Kim SH, Minata M, Mohyeldin A, Xie W, Chen D, et al: Targeting NEK2 attenuates glioblastoma growth and radioresistance by destabilizing histone methyltransferase EZH2. J Clin Invest 127: 3075-3089, 2017.

15. Wu Z, Lee ST, Qiao Y, Li Z, Lee PL, Lee YJ, Jiang X, Tan J, Aau M, Lim CZ and Yu Q: Polycomb protein EZH2 regulates cancer cell fate decision in response to DNA damage. Cell Death Differ 18: 1771-1779, 2011.

16. Vissers JH, van Lohuizen $M$ and Citterio E: The emerging role of Polycomb repressors in the response to DNA damage. J Cell Sci 125: 3939-3948, 2012.

17. Bouwman P and Jonkers J: The effects of deregulated DNA damage signalling on cancer chemotherapy response and resistance. Nat Rev Cancer 12: 587-598, 2012.

18. Yang Q, Nair S, Laknaur A, Ismail N, Diamond MP and Al-Hendy A: The polycomb group protein EZH2 impairs DNA damage repair gene expression in human uterine fibroids. Biol Reprod 94: 69, 2016.

19. Liu H, Li W, Yu X, Gao F, Duan Z, Ma X, Tan S, Yuan Y, Liu L, Wang J, et al: EZH2-mediated Puma gene repression regulates non-small cell lung cancer cell proliferation and cisplatin-induced apoptosis. Oncotarget 7: 56338-56354, 2016.

20. Zhou BB and Elledge SJ: The DNA damage response: Putting checkpoints in perspective. Nature 408: 433-439, 2000.

21. Yan S, Sorrell M and Berman Z: Functional interplay between ATM/ATR-mediated DNA damage response and DNA repair pathways in oxidative stress. Cell Mol Life Sci 71: 3951-3967, 2014.

22. Huang Y, Wang X, Niu X, Wang X, Jiang R, Xu T, Liu Y, Liang L, Ou X, Xing X, et al: EZH2 suppresses the nucleotide excision repair in nasopharyngeal carcinoma by silencing XPA gene. Mol Carcinog 56: 447-463, 2017.

23. Gursoy-Yuzugullu O, Carman C, Serafim RB, Myronakis M, Valente $\mathrm{V}$ and Price BD: Epigenetic therapy with inhibitors of histone methylation suppresses DNA damage signaling and increases glioma cell radiosensitivity. Oncotarget 8: 24518-24532, 2017.

24. Kirk JS, Schaarschuch K, Dalimov Z, Lasorsa E, Ku S, Ramakrishnan S, Hu Q, Azabdaftari G, Wang J, Pili R and Ellis L: Top2a identifies and provides epigenetic rationale for novel combination therapeutic strategies for aggressive prostate cancer. Oncotarget 6: 3136-3146, 2015.

25. Stamato MA, Juli G, Romeo E, Ronchetti D, Arbitrio M, Caracciolo D, Neri A, Tagliaferri P, Tassone P and Amodio N: Inhibition of EZH2 triggers the tumor suppressive miR-29b network in multiple myeloma. Oncotarget 8: 106527-106537, 2017.

26. Yu Q: Restoring p53-mediated apoptosis in cancer cells: New opportunities for cancer therapy. Drug Resist Updat 9: 19-25, 2006.

This work is licensed under a Creative Commons Attribution-NonCommercial-NoDerivatives 4.0 International (CC BY-NC-ND 4.0) License. 